\title{
The Salvo Combat Model with a Sequential Exchange of Fire ${ }^{1}$
}

\author{
Michael J Armstrong, Associate Professor \\ Goodman School of Business, Brock University, St Catharines, ON, L2S 3A1, Canada \\ michael.armstrong@brocku.ca
}

\begin{abstract}
This paper develops a version of the stochastic salvo combat model in which the exchange of fire is sequential, rather than simultaneous. This sequential-fire version is built by modifying the equations in the original simultaneous-fire version. The performance of the sequential model is tested by comparing its outputs to those of a Monte Carlo simulation. The fit between the model and the simulation is very close, especially for the mean and standard deviation of losses. The model is then applied to the Battle of the Coral Sea. The results suggest that attacking first would have given the American force a larger advantage than that provided by an extra aircraft carrier.
\end{abstract}

Keywords: Military, Defence, Simulation, Naval tactics, Salvo equations, Stochastic model.

\section{Introduction}

One way for a military force to seek an advantage over its opponent is to attack first. Historically, navies have achieved first strikes via strategic surprise (Pearl Harbour in 1941), superior scouting (Midway in 1942), tactical positioning (Philippine Sea in 1944), or longerranged weapons (Latakia in 1973). The potential benefits of striking first, and the potential costs of suffering such an attack, are of on-going interest to naval analysts (see, e.g., Lucas and McGunnigle, 2003; Tiah, 2007; Edmiston, 2011). It even has been argued that "attacking effectively first" should be a primary goal of naval tactics (Hughes, 2000: xxv).

The possibility of striking first has also been studied in other types of conflict, such as terrorist attacks. For example, some researchers have analysed game-theory models where terrorists choose which targets to attack, while government forces choose which targets to defend; see, for example, Zhuang and Bier (2007), He and Zhuang (2012), or Hausken and Zhuang (2012). In some of these attacker-defender games the attacking terrorists move first, while in other cases they move at the same time or after the defending government forces.

Given the importance of this factor in warfare past and present, it would be useful for analysts and historians to have a simple way to model missile combat where the two sides attack sequentially, instead of simultaneously. The salvo equations of Hughes (1995) already provide a simple way to represent combat involving missiles or airstrikes, much as the Lanchester models do for gunfire combat (see, e.g., MacKay, 2009; Johnson and MacKay, 2011; Atkinson et al,

\footnotetext{
${ }^{1}$ This is a post-peer-review, pre-copyedit version of an article published in the Journal of the Operational Research Society. The definitive publisher-authenticated version [Armstrong MJ, 2014. "The salvo combat model with a sequential exchange of fire", Journal of the Operational Research Society 65 \#10, 1593-1601, doi:10.1057/jors.2013.115] is available online at: http://www.palgrave-journals.com/jors/journal/v65/n10/full/jors2013115a.html
} 
2012). However, both the original deterministic salvo model and the later stochastic one (Armstrong, 2005) assume that the exchange of fire is simultaneous.

This paper modifies the salvo combat model to handle a sequential exchange of fire. In this version, one force executes its attack first while the opponent defends; subsequently, the opponent's survivors (if any) return fire while the original attacker defends.

The research begins with the deterministic salvo model (Hughes, 1995). Since this model is easily modified to handle sequential attacks, it allows an initial assessment of the value of attacking first, and of how that value is influenced by the combat units' characteristics.

The study then derives a sequential version of the stochastic salvo model (Armstrong, 2005). Since the salvo model is a highly simplified representation of combat, the modifications are chosen to maintain that simplicity as much as possible. The sequential version is tested by comparing its results to those of a Monte Carlo simulation, as in Armstrong (2011). The numerical results show that the model outputs fit very closely with the simulation outputs, especially for summary statistics such as the mean and variance of the losses.

The last part of the paper applies the sequential stochastic model to the 1942 Battle of the Coral Sea between American and Japanese aircraft carriers, as in Armstrong and Powell (2005). This counterfactual analysis suggests that the Americans could potentially have gained a large advantage if they had been able to attack before the Japanese, rather than at the same time. This first-strike advantage would have been more valuable than having an additional aircraft carrier. Conversely, being forced to attack second would likely have been fatal to the American task force and resulted in the loss of Port Moresby to the Japanese.

\section{The Basic Salvo Combat Model}

Consider a battle between two forces, Red and Blue. Let $A$ represent the number of combat units (e.g., warships) in the Red force at the beginning of the battle. Each one has offensive firepower $\alpha$, which is the number of missiles (or airstrikes, etc.) accurately fired per salvo at the enemy. Blue has $B$ units, each with defensive firepower $z$, which is the number of incoming enemy missiles intercepted per salvo by active defences. Each missile that is not intercepted causes the loss of portion $v$ of a Blue ship, so that the staying power $x=1 / v$ is the number of hits needed to incapacitate one Blue ship. Similar symbols represent Red's defensive firepower $y$, staying power $w$, and loss per hit $u$, along with Blue's offensive firepower $\beta$. See Table 1 for a summary of this notation.

The battle consists of Red firing a salvo of missiles that Blue tries to intercept, while Blue simultaneously launches a salvo that Red tries to intercept. Hughes (1995) developed the salvo equations to calculate the change in strength $\Delta B$ for Blue and $\triangle A$ for Red; the particular notation shown below is that of Armstrong (2005).

$$
\begin{array}{ll}
\Delta A=-(\beta B-y A) u & \text { subject to } 0 \leq-\Delta A \leq A \\
\Delta B=-(\alpha A-z B) v & \text { subject to } 0 \leq-\Delta B \leq B
\end{array}
$$


For example, Equation 1 first calculates Blue's total number of offensive missiles fired as $\beta B$, and then subtracts Red's total number of defensive interceptions as $y A$. If this difference is zero or less, i.e., if $(\beta B-y A) \leq 0$, this indicates that all of the missiles have been intercepted, and Red suffers no loss; hence $\Delta A=0$. If the difference instead is positive, $(\beta B-y A)>0$, this indicates the number of missiles that survive interception and hit Red ships; Red suffers loss $u$ for each such hit. Of course Red cannot lose more ships than it actually has, so its loss is capped at $-\triangle A=A$. The interested reader should see Hughes (1995) for further details about these equations, or Armstrong (2004) for an analysis of their mathematical properties.

\section{A Sequential Deterministic Model}

It is straightforward to modify the deterministic version (Hughes, 1995) of the salvo model to depict sequential fire. Several numerical studies have already used it that way (Lucas and McGunnigle, 2003; Tiah, 2007; Edmiston, 2011). Suppose that Blue attacks first while Red defends, and then the Red survivors (if any) return fire while Blue defends. With this sequence, Red suffers losses according to Equation 1 and is left with $A_{1}$ survivors as follows.

$$
A_{1}=A+\Delta A=A-(\beta B-y A) u=A-\beta u B+y u A \quad \text { subject to } 0 \leq A_{1} \leq A
$$

If $(\beta B-y A) u \geq A$, then $A_{1}=0$ and there is no Red return fire. Aside from that trivial case, the change in Blue's strength $\Delta B$ and its surviving force $B_{1}$ can be calculated as follows:

(a) If $\alpha(A-\beta u B+y u A) \leq z B$, then Red's return fire is all intercepted; thus $\Delta B=0$ and $B_{1}=B$.

(b) If $\alpha(A-\beta u B+y u A) \geq B(x+z)$, or equivalently $(\alpha y u+\alpha) /(\alpha \beta u+z+x) \geq B / A$, then Red's return fire completely eliminates Blue; thus $\Delta B=-B$ and $B_{1}=0$.

(c) Otherwise, Red's return fire eliminates part of Blue's force according to Equation 4.

$$
\Delta B=-\left(\alpha A_{1}-z B\right) v=-(\alpha(A-\beta u B+y u A)-z B) v=-(\alpha A-\alpha \beta u B+\alpha y u A-z B) v
$$

For example, suppose that Blue initially has $B=4$ ships while Red has $A=5$ ships. Let all ships be identical with offensive firepower $\alpha=\beta=4$ missiles per salvo, defensive firepower $y$ $=z=2$ interceptions per salvo, and loss per hit of $u=v=1 / 3$ ships. Blue's attack will eliminate $(4 \times 4-2 \times 5) / 3=2$ ships, leaving $5-2=3$ Red survivors. Those survivors will then return fire and cause Blue to lose $(4 \times 5-4 \times 2-4 \times 4 \times 4 / 3+4 \times 2 \times 5 / 3) / 3=1.33$ ships.

If Red could instead attack simultaneously, then Blue's losses would be $(4 \times 5-2 \times 4) / 3=4$ ships. Thus in this numerical example, shooting first greatly aids Blue by reducing its losses, while shooting second leaves Red relatively worse off for that same reason.

Not surprisingly, the desirability of shooting first and the corresponding undesirability of shooting second hold more generally in the salvo model. To be precise, shooting first is always at least as good as shooting simultaneously; and shooting simultaneously is always at least as good as shooting second. However, there are some special cases where different attack sequences provide the same outcomes, and thus are equally desirable. 
One such case occurs whenever either side is too weak to penetrate their opponent's defences; i.e., if $(\beta B-y A) \leq 0$ or $(\alpha A-z B) \leq 0$. In this case, the weaker side suffers the same loss regardless of the attack sequence, while the stronger side suffers no loss regardless of the attack sequence. For instance, this will always be true in low lethality battles (defined as having $\alpha / z \leq$ $y / \beta)$, because then at least one side is unable to hit the other (Armstrong, 2004). It can also be true in some high lethality battles (where $\alpha /(z+x) \geq(y+w) / \beta$ ) and moderate lethality battles (where $\alpha / z>y / \beta$ but $\alpha /(z+x)<(y+w) / \beta$ ), if one side outnumbers the other by such a wide margin that its defensive firepower can intercept all incoming fire.

Another partial special case can occur in moderate or high lethality battles if one side's offensive firepower is sufficient to eliminate its opponent even when shooting second. For example, this would apply for Red if $\alpha(A-\beta u B+y u A) \geq B(x+z)$. Red would then prefer to attack first and thereby avoid any losses itself; but it would be indifferent between attacking simultaneously and attacking second, because $\Delta B=-B$ and $\Delta A=-(\beta B-y A) u$ either way. Conversely, Blue would want to avoid attacking second, because it would be eliminated before it could fire; but it would be indifferent between attacking simultaneously and attacking first.

Returning to the previous numerical example, suppose that Red now has $A=8$ warships. Their defence could completely absorb any attack from Blue, as $(4 \times 4-2 \times 8) / 3=0$, and their offence would eliminate all the Blue ships. The outcome therefore would be the same regardless of who attacks first, making Red and Blue indifferent about the sequence.

In situations where none of these special cases holds, it can be informative to compare Blue's losses when firing first to those it suffers when firing simultaneously or second.

$$
\begin{aligned}
\Delta B_{1 s t} / \Delta B_{\text {Other }} & =(-(\alpha A-z B-\alpha u \beta B+\alpha u y A) v) /(-(\alpha A-z B) v) \\
& =1-(\alpha(\beta B-y A)) /(w(\alpha A-z B))
\end{aligned}
$$

The ratio on the right of this expression represents the relative reduction in Blue's losses due to attacking first. It can be shown that this benefit is increasing in Blue's strength $B$, offensive firepower $\beta$, and defensive firepower $z$. Conversely, the advantage is decreasing in Red's strength $A$, offense $\alpha$, defence $y$, and staying power $w$. For example, the effect of Red's offensive fire is found by taking the derivative of the ratio with respect to $\alpha$.

$$
\begin{aligned}
& \partial / \partial \alpha(\alpha(\beta B-y A)) /(w(\alpha A-z B)) \\
& =((\beta B-y A) w(\alpha A-z B)-(\alpha(\beta B-y A))(w A)) /\left(w^{2}(\alpha A-z B)^{2}\right) \\
& =(w(\beta B-y A)(\alpha A-z B-\alpha A)) /\left(w^{2}(\alpha A-z B)^{2}\right)=-(z B(\beta B-y A)) /\left(w(\alpha A-z B)^{2}\right)
\end{aligned}
$$

This derivative is negative when $(\beta B-y A)>0$ and $(\alpha A-z B)>0$, i.e., each side can hit the other.

Another way to assess the effect of attacking sequentially rather than simultaneously is via the fractional exchange ratio (FER). This ratio compares the fraction of Blue forces lost to the fraction of Red forces lost, as in FER $=(\Delta B / B) /(\triangle A / A)$. FER $=1$ indicates that both sides are losing the same proportion of their forces, so that the battle is a "tie" in some sense. The 
force ratio B/A where this happens is what Armstrong (2004) called the "parity point", and it provides one measure of the relative combat "worth" of the warships on each side.

To see this, consider again the previous numerical example. The Red and Blue ships have identical characteristics, so when attacking simultaneously they are clearly of equal value. FER $=1$ whenever both sides have the same number of ships, i.e., whenever $B / A=1$.

However, if Blue attacks first instead of simultaneously, then solving Equations 3 and 4 shows that FER $=1$ only when $B / A=0.573$. That is, each Red ship firing second is degraded to the equivalent of only 0.573 Blue ships firing first; or conversely, each first-firing ship becomes the equal of $1 / 0.573=1.74$ second-firing ships. This particular number is scenario-specific, but it provides a clear illustration of the "force multiplier" benefit of striking first.

\section{A Sequential Stochastic Model}

When modifying the stochastic version of the salvo model for sequential attacks, the main challenge is that the number of survivors who return fire is a random variable. This section describes one relatively straightforward way to handle that complication.

In the original stochastic salvo model (Armstrong, 2005), the number of accurate missiles $\alpha_{i}$ fired by each Red ship is treated as an independent and identically distributed random variable that follows a binomial distribution with mean $\mu_{\alpha}=n_{\alpha} p_{\alpha}$ and variance $\sigma \alpha^{2}=n_{\alpha} p_{\alpha}\left(1-p_{\alpha}\right)$. Here, $n_{\alpha}$ represents the number of missiles fired per ship, and $p_{\alpha}$ represents each one's probability of success. The number of interceptions $z_{j}$ by each Blue ship is likewise assumed to be binomial with parameters $\mu_{z}=n_{z} p_{z}$ and $\sigma_{z}^{2}=n_{z} p_{z}\left(1-p_{z}\right)$.

The total number of Blue's defensive interceptions $D e f_{B}$ is subtracted from the total number of Red's offensive missiles $O f f_{B}$ to get the net number of non-intercepted missiles $N_{e} t_{A B}$ $=O f f_{A}-D e f_{B}$. This is assumed to follow a normal distribution with cumulative distribution function (cdf) $F_{N e t A B}$ and probability density function (pdf) $f_{N e t A B}$. Each of these missiles hits a Blue ship and causes a random amount of damage $v_{k}$ with mean $\mu_{v}$ and variance $\sigma_{v}{ }^{2}$. The nominal number of Blue survivors $B_{1}$ * (i.e., before truncating the distribution's tails to account for $0 \leq-\Delta B \leq B$ ) follows a normal distribution with cdf $G_{B 1 *}$ and pdf $g_{B 1 *}$. Similar symbols represent Blue's attack and Red's defence. Table 2 contains a summary of this notation.

Suppose now that Blue attacks first. Equations 7 and 9 in Armstrong (2005) provide expressions for the mean $\mu_{\mathrm{A} 1}$ and variance $\sigma_{\mathrm{A} 1}^{2}$ of the number of Red survivors $A_{1}$; they are reproduced below. (The $\pm u_{v} / 2$ terms represent a continuity correction.) The interested reader should refer to that paper for the derivation of these equations.

$$
\begin{aligned}
\mu_{A 1}=\mu_{A 1^{*}}\left[F_{A 1^{*}}\left(A-\mu_{u} / 2\right)\right. & \left.-F_{A 1^{*}}\left(0+\mu_{u} / 2\right)\right]-\sigma_{A 1^{*}}^{2}\left[f_{A 1^{*}}\left(A-\mu_{u} / 2\right)-f_{A 1^{*}}\left(0+\mu_{u} / 2\right)\right] \\
+ & A\left(1-F_{A 1^{*}}\left(A-\mu_{u} / 2\right)\right)
\end{aligned}
$$




$$
\begin{aligned}
\sigma_{A 1}^{2} & =\left(\mu_{A 1^{*}}^{2}+\sigma_{A 1^{*}}{ }^{2}\right)\left[F_{A 1^{*}}\left(A-\mu_{u} / 2\right)-F_{A 1^{*}}\left(0+\mu_{u} / 2\right)\right]+A^{2}\left(1-F_{A 1^{*}}\left(A-\mu_{u} / 2\right)\right)-\left(E\left[A_{1}\right]\right)^{2} \\
& -\sigma_{A 1^{*}}{ }^{2}\left[\left(A-\mu_{u} / 2+\mu_{A 1^{*}}\right) f_{A 1^{*}}\left(A-\mu_{u} / 2\right)-\left(0+\mu_{u} / 2+\mu_{A 1^{*}}\right) f_{A 1^{*}}\left(0+\mu_{u} / 2\right)\right]
\end{aligned}
$$

In the sequential model, Red's return fire $O f f_{A l}$ consists of the total number of missiles fired by their survivors. It therefore is the sum of a random number $A_{1}$ of random variables $\alpha_{\mathrm{i}}$, with the following mean and variance (see, e.g., Ross, 1993: 94-98).

$$
\begin{gathered}
\mathrm{E}\left[O f f_{A 1}\right]=\mu_{\mathrm{A} 1} \mu_{\alpha} \\
\operatorname{Var}\left[O f f_{A 1}\right]=\mu_{\mathrm{A} 1} \sigma_{\alpha}^{2}+\mu_{\alpha}^{2} \sigma_{\mathrm{A} 1}^{2}
\end{gathered}
$$

Equation 9 is similar to the corresponding one in Armstrong (2005). In Equation 10, the second term reflects the added variation due to the randomness of Red's strength $A_{1}$.

Equations 9 and 10 for Red's attack are combined with existing ones from Armstrong (2005) for Blue's defence to get the nominal number of non-intercepted offensive missiles $\operatorname{Net}_{A B}$.

$$
\begin{aligned}
& \mathrm{E}\left[N e t_{A B}\right]=\mathrm{E}\left[O f f_{A 1}\right]-\mathrm{E}\left[D e f_{B}\right]=\mu_{\mathrm{A} 1} n_{\alpha} p_{\alpha}-B n_{z} p_{z} \\
& \operatorname{Var}\left[N e t_{A B}\right]=\operatorname{Var}\left[O f f_{A 1}\right]+\operatorname{Var}\left[D e f_{B}\right]=\mu_{\mathrm{A} 1} n_{\alpha} p_{\alpha}\left(1-p_{\alpha}\right)+n_{\alpha}^{2} p_{\alpha}^{2} \sigma_{\mathrm{A} 1}^{2}+B n_{z} p_{z}\left(1-p_{z}\right)
\end{aligned}
$$

Summing the random loss per missile $\mu_{v}$ over the random number of missiles Net $_{A B}$ leads to the following expressions for the nominal number of Blue survivors $B_{1} *$. These replace Equations 4 and 5 of Armstrong (2005).

$$
\begin{aligned}
& \mu_{B 1} *=B-E\left[N e t_{A B}\right] E[v]=B-\left(\mu_{\mathrm{A} 1} \mu_{\alpha}-B n_{z} p_{z}\right) \mu_{v} \\
& \sigma_{B 1 *}^{2}=\left(\mu_{\mathrm{A} 1} n_{\alpha} p_{\alpha}-B n_{z} p_{z}\right) \sigma_{v}^{2}+\left(\mu_{\mathrm{A} 1} n_{\alpha} p_{\alpha}\left(1-p_{\alpha}\right)+B n_{z} p_{z}\left(1-p_{z}\right)+\sigma_{A 1} n_{\alpha}^{2} p_{\alpha}^{2}\right) \mu_{v}^{2} \\
& -2 \sigma_{v}^{2}\left(\mu_{\mathrm{A} 1} n_{\alpha} p_{\alpha}-B n_{z} p_{z}\right) G_{N e t A B}(0)+2 \sigma_{v}^{2}\left(\mu_{\mathrm{A} 1} n_{\alpha} p_{\alpha}\left(1-p_{\alpha}\right)+B n_{z} p_{z}\left(1-p_{z}\right)\right. \\
& \left.+\sigma_{A l^{2}} n_{\alpha}^{2} p_{\alpha}^{2}\right) g_{N e t A B}(0)
\end{aligned}
$$

These expressions can be used to find the probability of various outcomes for Blue, such as the probability of no loss or of no survivors. They also lead to the mean and variance of the actual number of Blue survivors $B_{1}$, i.e., after accounting for $0 \leq B_{1} \leq B$.

$$
\begin{gathered}
P\left[B_{1}=B\right]=\left(1-G_{B 1^{*}}\left(B-\mu_{v} / 2\right)\right) \\
P\left[B_{1}=0\right]=G_{B 1^{*}}\left(0+\mu_{v} / 2\right) \\
E\left[B_{1}\right]=\mu_{B 1^{*}}\left[G_{B 1^{*}}\left(B-\mu_{v} / 2\right)-G_{B 1^{*}}\left(0+\mu_{v} / 2\right)\right]-\sigma_{B 1^{*}}{ }^{2}\left[g_{B 1^{*}}\left(B-\mu_{v} / 2\right)-g_{B 1^{*}}\left(0+\mu_{v} / 2\right)\right] \\
+B\left(1-G_{B 1^{*}}\left(B-\mu_{v} / 2\right)\right)
\end{gathered}
$$




$$
\begin{aligned}
\operatorname{Var}[ & \left.B_{1}\right]=\left(\mu_{B 1^{*}}{ }^{2}+\sigma_{B 1^{*}}{ }^{2}\right)\left[G_{B 1^{*}}\left(B-\mu_{v} / 2\right)-G_{B 1^{*}}\left(0+\mu_{v} / 2\right)\right] \\
& +B^{2}\left(1-G_{B 1^{*}}\left(B-\mu_{v} / 2\right)\right)-\left(E\left[B_{1}\right]\right)^{2} \\
& -\sigma_{B 1^{*}}{ }^{2}\left[\left(B-\mu_{v} / 2+\mu_{B 1^{*}}\right) g_{B 1^{*}}\left(B-\mu_{v} / 2\right)-\left(0+\mu_{v} / 2+\mu_{B 1^{*}}\right) g_{B 1^{*}}\left(0+\mu_{v} / 2\right)\right]
\end{aligned}
$$

This approach requires relatively few changes to the original simultaneous-fire equations and retains their ease of software implementation. However, it could be argued that it is overly simplistic, as the only information it uses about the number of Red survivors is the mean and variance. The shape of $A_{1}$ 's distribution is ignored, including the truncation of its tails at $0 \leq A_{1}$ $\leq A$. A more sophisticated approach could explicitly account for this truncation: e.g., if $A_{1}=0$, then there is no return fire; or if $A_{1}=A$, then every Red unit returns fire. Fortunately, the numerical testing in the next section suggests that such added complexity is unnecessary.

In this sequential stochastic model, the same preferences for attack sequence apply as in the deterministic case, but with two changes. Firstly, the preferences now need to be expressed in terms of averages or probabilities: e.g., shooting first is at least as good on average as shooting simultaneously, and shooting simultaneously is at least as good on average as shooting second. But in any given instance, a force that shoots second and is "lucky" could achieve better results than an identical force that shoots first but is "unlucky".

Secondly, the boundary cases for this sequence preference are gradual tendencies rather than distinct cut-offs. According to the deterministic model, a fleet that is much stronger or weaker than its opponent should be indifferent to the sequence of attacks, as the battle's outcome would be the same in any event. In the stochastic model, however, there is always a small chance that an inferior force could penetrate its opponent's defences with at least one missile. This suggests that even a greatly superior force should prefer to attack first, particularly if the leaders of the navy or its government are relatively risk-averse.

\section{Testing the Model with Monte Carlo Simulation}

This section compares the performance of the sequential-fire stochastic salvo model to that of a Monte Carlo simulation, much as Armstrong (2011) did with the simultaneous-fire version. The sequential salvo model (hereafter, "the model") is implemented in Excel spread sheet software, as in Armstrong (2007, 2011). The Monte Carlo simulation ("the simulation") is also set up in Excel, using the @ Risk software add-in. The same parameter inputs (scenarios) are used in both cases, and then their outputs (battle outcomes) are compared to see how closely they match. (The spread sheets are available from the author upon request.)

\section{Data Generation}

The input parameters for this numerical study are as follows. The size $B$ of the Blue force (which shoots first) is fixed at 4 warships, while the size $A$ of the Red force (which shoots second) is varied from 2 to 10 warships. These choices provide scenarios where the likely Blue 
outcome ranges from almost always being unharmed (when $A=2$ ), to almost always being eliminated (when $A=10$ ). For simplicity, all Red and Blue ships are identical: each can launch $n_{\alpha}=n_{\beta}=4$ offensive missiles and attempt $n_{y}=n_{z}=2$ defensive interceptions per salvo.

The probability of success for each offensive missile $p_{\alpha}=p_{\beta}$ is varied across 3 values: $0.50,0.67$, and 0.83 . The same 3 values are separately used for the probability of success for each defensive interception $p_{y}=p_{z}$. The damage caused by each missile is random with mean $\mu_{u}$ $=\mu_{v}=0.33$ ships and standard deviation $\sigma_{u}=\sigma_{v}=0.11$ ships; thus the average staying power is $1 / 0.33 \approx 3$ hits per ship. These are the same figures used in Armstrong $(2007,2011)$.

The combination of 9 Red forces sizes, 3 offensive missile probabilities, and 3 defensive interception probabilities gives a total of 81 scenarios for testing in both the model and the simulation. In terms of salvo combat lethality (Armstrong, 2004), these scenarios all fall into the moderate lethality category. The study avoids high lethality scenarios because their tendency towards heavy losses or "overkill" could overshadow any differences between the model and the simulation. The study also avoids low lethality scenarios, because if one side is effectively invulnerable, it does not matter whether it fires first or second.

For the simulation with @ Risk, the first half of the spread sheet calculates the losses inflicted by Blue against Red. A binomial distribution represents the number of accurate missiles from Blue, and another binomial distribution represents the number of successful interceptions by Red. The difference (if positive) between those two variables indicates the number of non-intercepted missiles that hit Red. For each missile, a normal distribution determines the loss suffered by Red. The second half of the spread sheet calculates the losses inflicted by the Red survivors (if any) against Blue in a similar manner.

The simulation tracks the Blue losses across 10000 trials (battles) for each of the 81 scenarios. Each scenario yields four metrics: the mean losses suffered by Blue, measured in ships; the standard deviation of those losses, in ships; the probability of losing zero ships; and the probability of losing all 4 ships. The mean and standard deviation are key summary statistics of the loss distribution, while the two probabilities examine the distribution's upper and lower tails.

\section{Data Analysis}

As an example of the results, consider the scenario where Red has 6 ships and the probability of success is 0.67 for each offensive missile and defensive interception. Across 10000 simulated trials, Blue losses had a mean of 2.637 ships and a standard deviation of 0.989 ships. Blue lost no ships in $0.8 \%$ of the trials, while it lost all of its ships in $12.5 \%$ of them.

By comparison, the model calculates Blue's mean loss to be 2.643 ships, or 0.006 ships higher than the simulation. The standard deviation is 0.986 ships, or 0.003 ships lower. The probability of losing zero ships is $1.1 \%$ and the probability of total loss is $15.2 \%$. Thus in this particular scenario, the fit between the model and the simulation is very close for the mean and standard deviation, and reasonably close for the tail probabilities. 
The next step is to assess the fit between the model and the simulation across all 81 scenarios at once. One qualitative approach uses scatter plots, as in Armstrong (2011). For example, in Figure 1 each circle represents one scenario; it matches the probability of Blue being totally eliminated as calculated by the model (vertical axis) with the corresponding probability from the simulation (horizontal axis). The diagram also includes a diagonal reference line to indicate where the dots hypothetically would fall if a perfect fit existed between model and simulation. In this figure most points fall just above the line, indicating that the model tends to slightly overestimate this probability, especially for values near 0.5 . This may be due to the simple continuity correction Armstrong (2005) used in the model, which assigns probability mass near zero to zero. Scatter plots for the other 3 metrics (not shown) suggest even closer fits.

To confirm these visual impressions, linear regressions were calculated next for each of the 4 metrics across all 81 pairs of measurements, with the regression constants fixed to zero. The top half of Table 3 displays the results. In this context, slope coefficients and $R^{2}$ values close to 1.000 indicate that the model outputs are similar to the simulation outputs.

For example, regression of the probabilities of complete elimination from the model against those of the simulation yielded $R^{2}=0.999$, showing that the model gives extremely close estimates of the simulation values; it explains $99.9 \%$ of the variation in them. The slope coefficient of 1.026 meanwhile confirms that the model tends to marginally overestimate these probabilities. The results for the other 3 metrics indicate fits that are at least as good.

The bottom half of the table quantifies the model-to-simulation fit in a different way. First, the difference between each result from the model and the equivalent result from the simulation was calculated, as in, e.g., (difference in mean loss $)=($ model mean loss $)-$ (simulation mean loss). Then the averages and variances of those differences across all 81 scenarios were determined. Average differences close to zero indicate that the model gives an accurate (unbiased) estimate of the simulation, whereas positive averages indicate a tendency to overestimate and negative averages a tendency to underestimate. Similarly, variances close to zero suggest that the model is consistent in its estimates of the simulation, whereas larger variances suggest wider swings from scenario to scenario. The table also shows the difference with the largest magnitude, positive or negative. All of these figures confirm that the fit between the model and the simulation is generally very close.

\section{Applying the Model to a Carrier Battle}

This section demonstrates the application of the sequential stochastic salvo model in a small study of the Battle of the Coral Sea (the Monte Carlo simulation is not used in this part of the study). This battle was important historically because it was the first naval engagement fought entirely via an exchange of carrier airstrikes, and strategically because it saved Port Moresby from Japanese invasion. At the tactical level, the losses were heavier on the American side: the Lexington sank and the Yorktown needed major repairs in Pearl Harbor. On the Japanese side, the crippled Shokaku required months to repair; the Zuikaku remained operational, but returned to Japan to replenish its aircraft (Lundstrom, 1984). 
In the battle on 8 May 1942, the attacks between the aircraft carriers (CVs) of the United States Navy (USN) and the Imperial Japanese Navy (IJN) were effectively simultaneous. But they could easily have been sequential if, as at Midway, one fleet had spotted the other sooner.

This work extends an earlier counterfactual study by Armstrong and Powell (2005). They varied the number of USN CVs, the disposition of those CVs (grouped or dispersed), and their ratio of defensive fighters to offensive bombers. Their results indicate that adding another USN CV would have substantially increased IJN losses, but only marginally decreased USN losses. As well, the USN's outcome would likely have been better if it had dispersed its CVs, but worse if it had carried more fighters and fewer bombers.

The new factor to vary here is the sequence of attacks; three possibilities are compared.

- Simultaneous: The USN and IJN conduct their airstrikes simultaneously, as in the actual battle, and as in Armstrong and Powell (2005).

- USN first: The USN executes its attack first while the IJN defends; then the IJN survivors respond with a counter-attack while the USN defends.

- USN second: The IJN executes its attack first while the USN defends; then the USN survivors respond with a counter-attack while the USN defends.

The other parameter that is varied here is the number of American carriers. Historically the USN had $2 \mathrm{CVs}$ in the battle, but this study also tries value of 1,3 , and 4 , as there were 4 USN CVs in the Pacific theatre at that time. Combining the 4 levels of USN force strength along with the 3 attack sequences yields 12 different scenarios for comparison.

The model inputs for these scenarios are shown in Table 4. They were derived in Armstrong and Powell (2005) using historical data. Each point of offensive firepower represents one dive bomber or torpedo bomber, and each point of defensive firepower represents one fighter aircraft. For example, each USN CV carries 17 fighters, and each of those fighters has a probability of 0.3529 of successfully intercepting one IJN bomber.

Table 5 contains the main model outputs for both the USN and IJN, rounded to two decimal places. These indicate the average $\mathrm{CV}$ losses, the standard deviation of those losses, the probability of losing zero CVs, and the probability of losing all of the CVs. The final column subtracts the mean USN loss from the mean IJN loss; a positive number indicates an American advantage, while a negative one indicates a Japanese advantage.

For example, the fourth row of the table describes the historical case where the USN has $2 \mathrm{CVs}$ and attacks simultaneously with the IJN; these numbers are the same as in Armstrong and Powell (2005). The model estimates the average number of CVs incapacitated (not necessarily sunk) at 1.82 for the USN and 1.44 for the IJN. These are reasonably similar to, though perhaps slightly higher than, the actual results of the battle. The difference of the means, $1.44-1.82=-$ $0.38 \mathrm{CVs}$, can be seen as fairly representing the tactical defeat suffered by the USN.

By comparison, the fifth row of Table 5 shows the results of the same USN force attacking first. The USN's average loss decreases from 1.82 to just $0.17 \mathrm{CVs}$, while the 
standard deviation remains relatively unchanged at 0.18 versus $0.19 \mathrm{CVs}$. The probability of the American force suffering no loss at all jumps from $0 \%$ to $38.2 \%$, while the probability of being completely destroyed drops from $30.6 \%$ to $0 \%$. The IJN loss statistics remain unchanged, so the difference in the average losses changes from - 0.38 (a small American disadvantage) to +1.27 (a large American advantage).

Looking down the rest of the table, the numbers show that attacking first greatly reduces the USN's losses whenever it employs 2 or more CVs. In this context, striking first is more valuable than adding another CV. This point is illustrated in Figure 2, which shows the difference in mean losses from the model for each combination of carrier strength and attack type. While attacking first almost always provides some advantage to the USN, there is clearly an interaction effect between the sequence of attacks and the number of carriers. The incremental benefit of having an extra CV is greatest when the USN attack firsts; or to put it another way, the benefit of a first strike is greatest when there are enough CVs to deliver a decisive blow.

By contrast, a lone USN CV receives little benefit from attacking first, as it lacks enough firepower to fight 2 opponents. To "attack effectively first" (Hughes, 2000: xxv), one must not only attack first, but also attack effectively. The plot also illustrates that "more" is not always "better". If the USN attacks simultaneously or second, they actually get better average results with $1 \mathrm{CV}$ than with 2 , because the latter choice exposes more of their ships to loss.

These results suggest that if the USN had actually been able to attack first with its $2 \mathrm{CVs}$, the battle's tactical outcome would likely have been much more favourable. The Lexington would not have sunk and the Yorktown would have been untouched. This would have given the USN an extra margin of safety for the Battle of Midway one month later.

Conversely, attacking second would likely have been disastrous for the USN. The minimally damaged IJN CVs could have launched a follow-up airstrike against the weakened USN. The Japanese would thereby have established control of the Coral Sea and been able to complete their invasion of Port Moresby.

\section{Discussion}

This paper develops a sequential-fire version of the stochastic salvo combat model by modifying the existing simultaneous-fire model. This modified model allows researchers to quantify the benefits of striking first, and yet it remains simple enough to implement in userfriendly software such as spread sheets. Numerical tests indicate that the model produces results very similar to those of Monte Carlo simulation.

The model is then used to study the impact of different attack sequences in the 1942 Battle of the Coral Sea. The results indicate that an American first strike would have been more beneficial for them than having another aircraft carrier but striking simultaneously. Conversely, a Japanese first strike would have been devastating for the Americans and allowed the Japanese to invade Port Moresby. 
Future mathematical research could explore more sophisticated sequential models to see whether additional complexity would provide any meaningful improvement in accuracy. Future research could also examine situations where each side may be able to influence (perhaps at some cost) the sequence of attacks; this would involve adding elements of game theory, as in the attacker-defender game literature.

On the empirical side, researchers could use the new model to study the naval battles at Midway in 1942 and the Philippine Sea in 1944, where the airstrikes did occur sequentially. The model could also be adapted for other contexts, such as Connors' (2012) study of the 1853 Battle of Balaclava or Edmiston's (2011) study of a hypothetical near-future battle. Given the ample empirical data that is available for the 1944 Ardennes campaign, there might be potential for study there as well (see, e.g., Hausken and Moxnes, 2005).

\section{References}

Armstrong MJ (2011). A verification study of the stochastic salvo combat model. Annals of Operations Research 186 (1): 23-38.

Armstrong MJ (2007). Effective attacks in the salvo combat model: salvo sizes and quantities of targets. Naval Research Logistics 54 (1): 66-77.

Armstrong MJ (2005). A stochastic salvo model for naval surface combat. Operations Research 53 (5): 830-841.

Armstrong MJ (2004). Effects of lethality on naval combat models. Naval Research Logistics 51 (1): $28-43$.

Armstrong MJ and Powell MB (2005). A salvo combat analysis of the Battle of the Coral Sea. Military Operations Research 10 (4): 27-38.

Atkinson MP, Gutfraind A, Kress M (2012). When do armed revolts succeed: lessons from Lanchester theory. The Journal of the Operational Research Society 63 (10): 1363-1373.

Connors D (2012). Into the Valley of Death Rode the Digital Six Hundred. Major research paper, Department of History, Brock University, St Catharines.

Edmiston R (2011). Palawan Scenario: Hughes Salvo and Gaining Sea Superiority. Campaign analysis paper, Operations Research Department, US Naval Postgraduate School, Monterey.

Hausken K and Moxnes JF (2005). Approximations and empirics for stochastic war equations. Naval Research Logistics 52: 682-700.

Hausken K and Zhuang J (2012). The timing and deterrence of terrorist attacks due to exogenous dynamics. Journal of the Operational Research Society 63 (6): 726-735. 
He F and Zhuang J (2012). Modeling 'Contracts' between Terrorist Groups and Governments in a Sequential Game. Journal of the Operational Research Society 63 (6): 790-809.

Hughes WP (2000). Fleet Tactics and Coastal Combat, 2nd Ed. Naval Institute Press, Annapolis.

Hughes WP (1995). A salvo model of warships in missile combat used to evaluate their staying power. Naval Research Logistics 42 (2): 267-289.

Johnson IR and MacKay NJ (2011). Lanchester models and the Battle of Britain. Naval Research Logistics 58: 210-222.

Lucas TW and McGunnigle JE (2003). When is model complexity too much? Illustrating the benefits of simple models with Hughes' salvo equations. Naval Research Logistics 50: 197-217.

Lundstrom JB (1984). The First Team: Pacific Naval Air Combat from Pearl Harbor to Midway, Naval Institute Press, Annapolis.

MacKay NJ (2009). Lanchester models for mixed forces with semi-dynamical target allocation. Journal of the Operational Research Society 60: 1421-1427

Ross SM (1993). Probability Models, 5th ed. Academic Press, San Diego.

Tiah YM (2007). An Analysis of Small Navy Tactics Using A Modified Hughes' Salvo Model. Master's thesis, Operations Research Department, US Naval Postgraduate School, Monterey.

Zhuang J and Bier VM (2007). Balancing terrorism and natural disasters - defensive strategy with endogenous attack effort. Operations Research 55 (5): 976-991. 
Table 1: Notation for deterministic model

\begin{tabular}{lll}
\hline Red & Blue & Description \\
\hline$A$ & $B$ & Beginning force strength \\
$\alpha$ & $\beta$ & Offensive power per unit \\
$u$ & $v$ & Loss suffered per hit \\
$w$ & $x$ & Staying power per unit \\
$y$ & $z$ & Defensive power per unit \\
\hline
\end{tabular}

Table 2: Additional notation for stochastic model

\begin{tabular}{lll}
\hline Red & Blue & Description \\
\hline$n_{\alpha}$ & $n_{\beta}$ & Offensive missiles per unit per salvo \\
$p_{\alpha}$ & $p_{\beta}$ & Probability of an accurate missile \\
$O f f_{A}$ & $O f f_{B}$ & Total accurate offensive missiles per salvo \\
$n_{y}$ & $n_{z}$ & Defensive interceptions per unit per salvo \\
$p_{y}$ & $p_{z}$ & Probability of a successful interception \\
Def $_{A}$ & $D_{e} f_{B}$ & Total successful defensive interceptions per salvo \\
$N_{\text {et }} B_{B A}$ & Net $_{A B}$ & Nominal non-intercepted offensive missiles \\
$F_{N e t B A}$ & $G_{N e t A B}$ & cdf of nominal non-intercepted offensive missiles \\
$f_{N e t B A}$ & $g_{N e t A B}$ & pdf of nominal non-intercepted offensive missiles \\
$A_{1} *$ & $B_{1} *$ & Nominal surviving force strength after one salvo \\
$F_{B I^{*}}$ & $G_{B I^{*}}$ & cdf of nominal surviving force strength \\
$f_{B I^{*}}$ & $g_{B 1^{*}}$ & pdf of nominal surviving force strength \\
$A_{I}$ & $B_{1}$ & Actual surviving force strength after one salvo \\
\hline
\end{tabular}

Table 3: Comparison of Blue losses, model versus simulation, for mean loss, standard deviation of loss (SD), probability of zero loss (P[0]), and probability of total loss (P[all])

\begin{tabular}{lcrrr}
\hline & Mean & \multicolumn{1}{c}{ SD } & P[0] & P[all] \\
\hline Regression $R^{2}$, model v. simulation & 1.000 & 0.999 & 1.000 & 0.999 \\
Regression slope, model v. simulation & 1.000 & 0.996 & 1.000 & 1.026 \\
Average of differences, model - simulation & 0.001 & -0.005 & 0.001 & 0.014 \\
Variance of differences, model - simulation & 0.000 & 0.000 & 0.000 & 0.000 \\
Greatest difference, model - simulation & 0.059 & -0.052 & -0.012 & 0.057 \\
\hline
\end{tabular}


Table 4: Coral Sea model inputs derived in Armstrong \& Powell (2005)

\begin{tabular}{lrr}
\hline & USN & \multicolumn{1}{c}{ IJN } \\
\hline Number of aircraft carriers & 2 & 2 \\
Bomber aircraft per carrier & 47 & 33 \\
Fighter aircraft per carrier & 17 & 20.5 \\
Probability of successful deployment per bomber & 0.5000 & 0.7727 \\
Probability of successful intercept per fighter & 0.35290 & 0.04878 \\
Average loss suffered per bomber & 0.04766 & 0.03208 \\
Standard deviation of loss per bomber & 0.01589 & 0.01069 \\
\hline
\end{tabular}

Table 5: Estimated Coral Sea losses from the model as the attack sequence and USN force size are varied. The numbers show the mean loss, standard deviation of loss (SD), probability of zero loss $(\mathrm{P}[0])$, and probability of total loss $(\mathrm{P}[\mathrm{all}])$, as well as the difference of means $=(\mathrm{USN}$ mean) - (IJN mean).

\begin{tabular}{|c|c|c|c|c|c|c|c|c|c|c|}
\hline $\begin{array}{l}\text { USN } \\
\text { CVs }\end{array}$ & $\begin{array}{c}\text { Attack } \\
\text { Sequence }\end{array}$ & $\begin{array}{l}\text { USN: } \\
\text { Mean }\end{array}$ & SD & $\mathrm{P}[0]$ & $\mathrm{P}[\mathrm{All}]$ & $\begin{array}{l}\text { IJN: } \\
\text { Mean }\end{array}$ & $\mathrm{SD}$ & $\mathrm{P}[0]$ & $\mathrm{P}[\mathrm{All}]$ & $\begin{array}{c}\text { Difference } \\
\text { of Means }\end{array}$ \\
\hline 1 & Simultaneous & 1.00 & 0.00 & $0 \%$ & $100 \%$ & 0.69 & 0.13 & $0 \%$ & $0 \%$ & -0.31 \\
\hline 1 & USN first & 0.99 & 0.05 & $0 \%$ & $92 \%$ & 0.69 & 0.13 & $0 \%$ & $0 \%$ & -0.30 \\
\hline 1 & USN second & 1.00 & 0.00 & $0 \%$ & $100 \%$ & 0.00 & 0.01 & $96 \%$ & $0 \%$ & -1.00 \\
\hline 2 & Simultaneous & 1.82 & 0.18 & $0 \%$ & $31 \%$ & 1.44 & 0.18 & $0 \%$ & $0 \%$ & -0.38 \\
\hline 2 & USN first & 0.17 & 0.19 & $38 \%$ & $0 \%$ & 1.44 & 0.18 & $0 \%$ & $0 \%$ & +1.27 \\
\hline 2 & USN second & 1.82 & 0.18 & $0 \%$ & $31 \%$ & 0.10 & 0.11 & $36 \%$ & $0 \%$ & -1.72 \\
\hline 3 & Simultaneous & 1.57 & 0.25 & $0 \%$ & $0 \%$ & 1.98 & 0.06 & $0 \%$ & $84 \%$ & +0.41 \\
\hline 3 & USN first & 0.00 & 0.00 & $100 \%$ & $0 \%$ & 1.98 & 0.06 & $0 \%$ & $84 \%$ & +1.98 \\
\hline 3 & USN second & 1.57 & 0.25 & $0 \%$ & $0 \%$ & 1.01 & 0.24 & $0 \%$ & $0 \%$ & -0.56 \\
\hline 4 & Simultaneous & 1.29 & 0.26 & $0 \%$ & $0 \%$ & 2.00 & 0.01 & $0 \%$ & $100 \%$ & +0.71 \\
\hline 4 & USN first & 0.00 & 0.00 & $100 \%$ & $0 \%$ & 2.00 & 0.01 & $0 \%$ & $100 \%$ & +2.00 \\
\hline 4 & USN second & 1.29 & 0.26 & $0 \%$ & $0 \%$ & 1.88 & 0.17 & $0 \%$ & $50 \%$ & +0.59 \\
\hline
\end{tabular}


Figure 1: Scatter plot comparing model versus simulation for the probability that all Blue units are eliminated. Each circle represents the results from one scenario, while the diagonal line indicates where the circles would fall if the fit had been perfect.

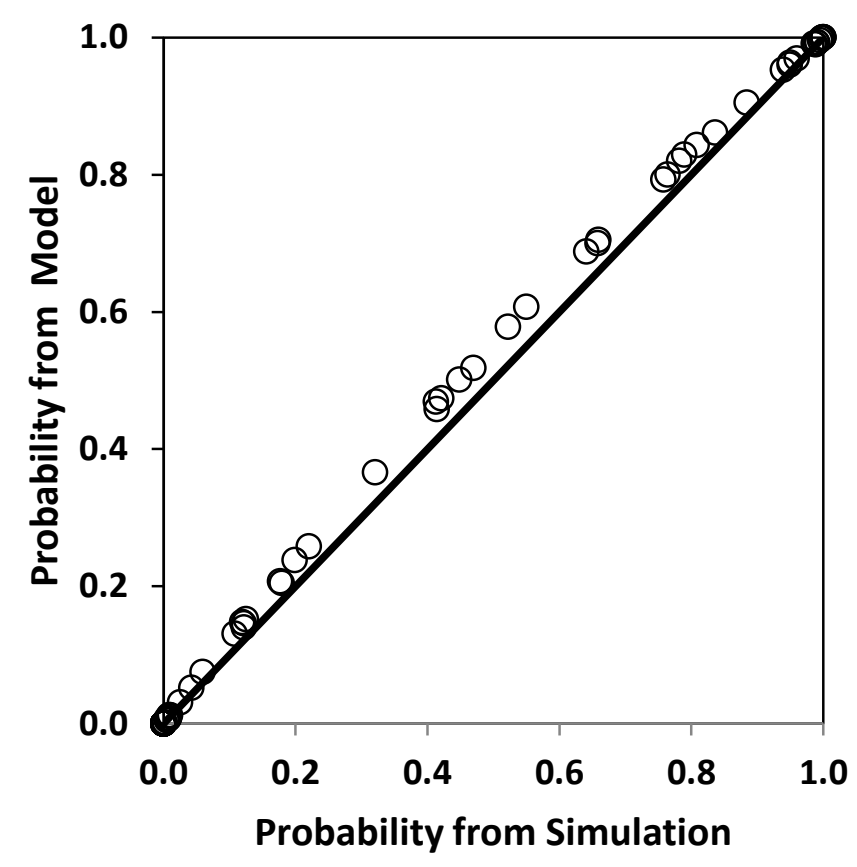

Figure 2: Interaction plot for the difference between the IJN and USN mean losses from the model as the attack sequence and the USN force size are varied. Positive numbers indicate a USN advantage.

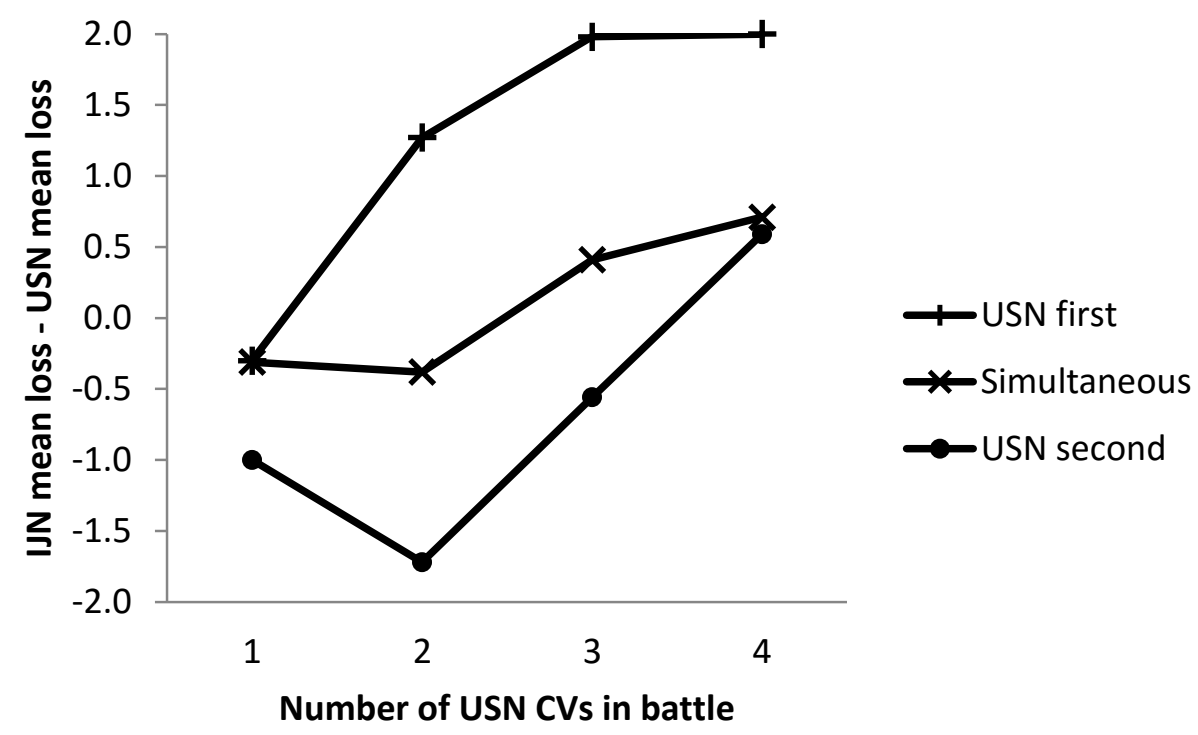

